\title{
Promoter recognition by bacterial alternative $\sigma$ factors: the price of high selectivity?
}

\begin{abstract}
Andrey Feklistov and Seth A. Darst ${ }^{1}$
The Rockefeller University, New York, New York 10065, USA

A key step in bacterial transcription initiation is melting of the double-stranded promoter DNA by the RNA polymerase holoenzyme. Primary $\sigma$ factors mediate the melting of thousands of promoters through a conserved set of aromatic amino acids. Alternative $\sigma s$, which direct transcription of restricted regulons, lack the full set of melting residues. In this issue of Genes \& Development, Koo and colleagues (pp. 2426-2436) show that introducing the primary $\sigma$ melting residues into alternative os relaxes their promoter specificity, pointing to a tradeoff of reduced promoter melting capacity for increased promoter stringency.
\end{abstract}

Transcription initiation in bacteria is controlled primarily by $\sigma$ factors, promoter specificity subunits for the catalytic core RNA polymerase (RNAP) (Gruber and Gross 2003; Paget and Helmann 2003). The primary $\sigma$ $\left(\sigma^{70}\right.$ in Escherichia coli) directs core RNAP to the majority of promoters active during log-phase growth. Alternative $\sigma$ s control restricted, specialized regulons in response to environmental conditions. Examples in E. coli (Eco) include $\sigma^{\mathrm{S}}$ (general stress response, stationary phase) (Klauck et al. 2007), $\sigma^{32}$ (also called $\sigma^{\mathrm{H}}$; cytoplasmic heat shock) (Guisbert et al. 2008), $\sigma^{28}$ (also called $\sigma^{\mathrm{F}}$; starvation, flagellar synthesis) (Chilcott and Hughes 2000), $\sigma^{\mathrm{E}}$ (envelope stress response) (Ades 2008), and FecI (iron deficiency) (Braun et al. 2003). Some bacteria have just one $\sigma$ factor (Mycoplasma sp.), while some have at least 66 (Streptomyces coelicolor). The vast majority of $\sigma$ factors, including all those described above, comprise a homologous family of proteins, the $\sigma^{70}$ family (Stragier et al. 1985; Gribskov and Burgess 1986), with four regions of conserved amino acid sequence (Lonetto et al. 1992; Gruber and Bryant 1997).

After binding to the core RNAP, the $\sigma$ factor directs the resulting holoenzyme to a particular set of promoters dictated by sequence-specific recognition of the promoter

[Keywords: $\sigma$ factor; Region 2.3; melting proficiency; promoter stringency]

${ }^{1}$ Corresponding author.

E-MAIL darst@rockefeller.edu; FAX (212) 327-7477.

Article is online at http://www.genesdev.org/cgi/doi/10.1101/gad.1862609. by $\sigma$ (Murakami et al. 2002; Gruber and Gross 2003). Promoter motifs recognized by $\sigma^{70}$ family members include (from upstream to downstream) the -35 element (Gardella et al. 1989; Siegele et al. 1989; Campbell et al. 2002), the extended -10 element (Keilty and Rosenberg 1987; Barne et al. 1997; Koo et al. 2009a,b), the -10 element (Siegele et al. 1989; Daniels et al. 1990; Waldburger et al. 1990; Tatti et al. 1991), and the discriminator (Feklistov et al. 2006; Haugen et al. 2006). Primary os, such as $\sigma^{70}$, recognize thousands of promoters constructed from subsets of these motifs that are poorly constrained in sequence. In contrast, alternative $\sigma$ s recognize only a handful of promoters-for example, Eco $\sigma^{32}$ directs the transcription of $\sim 50$ promoters, while $E c O \sigma^{28}$ transcribes $\sim 25$ promoters (Nonaka et al. 2006; Shen et al. 2006) - and these promoters require good matches to the cognate -35 , extended -10 , and -10 motifs (Koo et al. 2009a,b,c). In other words, primary $\sigma$ s tolerate a great deal of promoter sequence diversity (loose stringency), while alternative $\sigma$ s in general do not (high stringency). What are the sequence determinants of the $\sigma$ factors that govern this dramatic range of promoter stringency, and what are the mechanistic underpinnings? In this issue of Genes \& Development, Koo et al. (2009c) set out to answer these questions.

For $\sigma$ factors belonging to the $\sigma^{70}$ family, recognition of the promoter sequence is followed spontaneously by a series of isomerization steps yielding the transcription-competent open promoter complex (Fig. 1A), in which $\sim 14$ base pairs of DNA are melted in a region that includes most of the -10 element and extends downstream to the transcription start site (deHaseth et al. 1998). Studies on primary $\sigma$ s indicate that $\sigma$ plays a key role in the initiation of the DNA melting process within the promoter -10 element. A set of four aromatic residues that are invariant in primary $\sigma \mathrm{s}$, corresponding to Eco $\sigma^{70}$ F427, Y430, W433, and W434 (Gruber and Bryant 1997; Campbell et al. 2002), have been implicated in this promoter melting function (Helmann and Chamberlin 1988; Juang and Helmann 1994, 1995), possibly by interacting with the exposed bases of the nontemplate strand within the -10 element at the upstream edge of the transcription bubble to stabilize the melted 


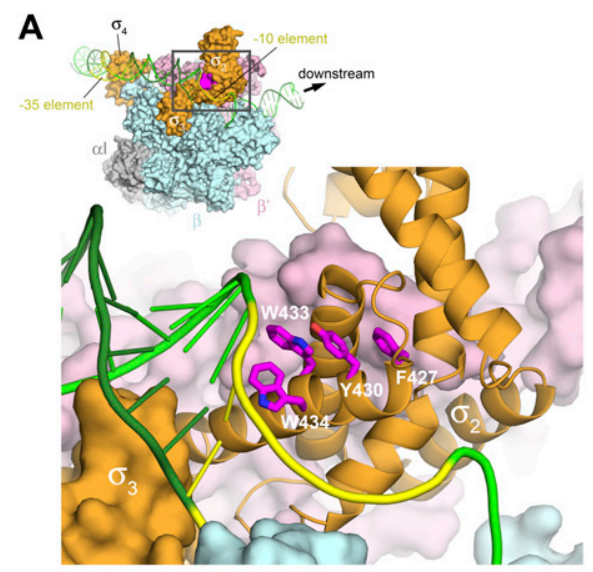

B

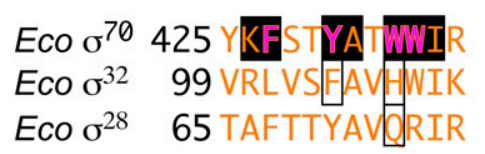

Figure 1. Primary $\sigma$ promoter melting residues in the context of the RNAP holoenzyme open promoter complex. (A) Structural model of the RNAP holoenzyme open promoter complex (after Murakami et al. 2002). The RNAP is shown as a molecular

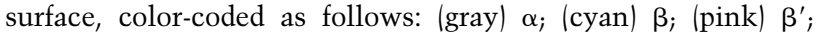
(orange) $\sigma$. The DNA is shown as phosphate backbone ribbons (template strand, dark green; nontemplate strand, light green), with the -35 and -10 elements colored yellow. The direction of transcription (downstream) is to the right. The top left (inset) shows an overview of the entire complex. The region in the gray box is magnified below. In the magnified view, $\sigma$ domain $2\left(\sigma_{2}\right)$ is shown as a backbone ribbon. The side chains of the four, universally conserved "promoter melting" mutants of primary $\sigma s$ (see the text) are shown in magenta. The labels show residue numering according to Eco $\sigma^{70}$. (B) Sequence context of the promoter melting residues. The "promoter melting" residues of the primary $\sigma$ are bold and colored magenta. Absolutely conserved residues of the primary $\sigma$ s are shaded with a solid black box. The corresponding residues of the alternative $\sigma s$ that were substituted by Koo et al. (2009c) are boxed.

state of the DNA (Roberts and Roberts 1996; Huang et al. 1997; Marr and Roberts 1997). Structural studies of an RNAP holoenzyme/promoter fragment complex are consistent with this view (Fig. 1A; Murakami et al. 2002). Despite significant sequence variation, the structures of the conserved domains of primary and alternative $\sigma$ s are essentially identical (Malhotra et al. 1996; Campbell et al. 2002, 2003, 2007; Li et al. 2002; Sorenson et al. 2004), and their interactions with core RNAP and with promoter DNA are expected to be conserved. Nevertheless, the crucial "promoter melting" residues of the primary $\sigma$ s are not shared among the alternative $\sigma$ s (Lonetto et al. 1992).

The study by Koo et al. (2009c) focuses on two Eco alternative $\sigma$ factors: $\sigma^{32}$ and $\sigma^{28}$ (encoded by rpoH and $r p o F$, respectively). $\sigma^{32}$ is induced in response to the presence of unfolded proteins in the cytoplasm, which can result from heat shock or other stressful conditions. $\sigma^{32}$ elicits transcription of regulons resulting in the expression of chaperones and proteases helping to refold or degrade damaged proteins (transcription of $\sim 90$ genes is initiated; e.g., dnaK, groESL, and hslU) (Nonaka et al. 2006). $\sigma^{28}$ directs transcription from $\sim 25$ promoters controlling flagellin biosynthesis and chemotaxis (Chilcott and Hughes 2000).

The "promiscuous" primary $\sigma, \sigma^{70}$, recognizes an extended $-10 /-10$ element consensus of TGnTATAAT (Pribnow 1975; Shultzaberger et al. 2006). The extended -10 motif (TG) is recognized by $\sigma$ domain 3 (Barne et al. 1997), while the -10 element is recognized by $\sigma$ domain 2, which contains the "melting" residues F427, Y430, $\mathrm{W} 433$, and W434. $\sigma^{32}$ and $\sigma^{28}$ recognize -10 elements of CCCCATnT and GCCGATAA, respectively. Previous studies by Koo et al. $(2009 \mathrm{a}, \mathrm{b})$ showed that these $\sigma^{32}$ and $\sigma^{28}-10$ elements are, in fact, composite, and their recognition follows a general bipartite pattern similar to $\sigma^{70}$, with an extended -10 element (upstream CC for $\sigma^{32}$, GC for $\sigma^{28}$ ) recognized by domain 3 , and a -10 element recognized by residues in region 2 .

The very first step in promoter opening probably involves flipping out of the consensus adenine residue, dubbed the "master base in promoter opening" (the $-11 \mathrm{~A}$, the second adenine in the TATAAT sequence), from the DNA duplex into a hydrophobic pocket in $\sigma$ (Lim et al. 2001). This triggers recognition of the remaining bases of the -10 element, which is followed by extension of the melted region to the transcription start site. The universally conserved primary $\sigma$ "melting residues" appear to act by promoting the $-11 \mathrm{~A}$ flipping, and in sequence-specific recognition of the -10 element nontemplate strand bases following the melting, with Y430 and W433 being the most crucial for the nucleation process (Tomsic et al. 2001; Schroeder et al. 2009). Using a combined in vivo/in vitro approach, Koo et al. (2009c) investigated the effects of substituting amino acid residues in the alternative $\sigma \mathrm{s}$ at positions corresponding to $\sigma^{70} \mathrm{Y} 430$ and W433 to those present in primary $\sigma \mathrm{s}$ (Fig. 1B). These substitutions rendered the alternative $\sigma s$ more promiscuous, in that they were able to tolerate promoters with less than the normally required complete set of promoter motifs (-35/extended $-10 /-10$ elements), with nonoptimal motif sequences, and with nonoptimal spacing between the elements.

The principal finding of the Koo et al. (2009c) study comes from the results of in vitro binding assays comparing $\sigma$ binding to promoter templates made of dsDNA with so-called fork junction templates /Guo and Gralla 1998). Fork junction DNA, which contains a doublestranded -35 element but mostly only the nontemplate strand of the -10 element, forms stable complexes with the RNAP holoenzyme that mimic many properties of the promoter open complex (Tsujikawa et al. 2001; Murakami et al. 2002). Thus, comparison of RNAP holoenzyme binding to a double-stranded versus a fork junction promoter relates to the stability of the initial, closed promoter complex versus the melted, open promoter complex. Koo et al. (2009c) observed that the mutated alternative $\sigma$ s were much more proficient then their wildtype counterparts in forming fork junction promoter complexes relative to double-stranded promoter complexes. 
The investigators concluded that the primary $\sigma$ "melting residues," placed in the context of the alternative $\sigma \mathrm{s}$, made the mutant alternative $\sigma$ s more efficient in the DNA strand separation (melting) step of promoter utilization, and that this was also the explanation for the increased tolerance toward imperfect promoters.

One can think of an evolutionary trade-off between melting capacity of a $\sigma$ factor and its promoter specificity. A $\sigma$ factor with strong melting capability would serve as a global regulator, serving many, poorly conserved promoters. $\sigma$ Factors with weak melting capability would act as local regulators, confined to a more restricted set of promoters. In a broad sense, the results of Koo et al. (2009c) provide an interesting corollary to the recent observation that transcription factors with high DNAbinding specificity tend to mediate a more focused response on a genome-wide scale, while lower DNA-binding specificity enables them to control a broad range of targets in a global manner (Lozada-Chavez et al. 2008).

The region of $\sigma$ harboring the "melting residues" $/ \sigma^{70}$ F427, Y430, W433, and W434), the so-called $\sigma$ conserved region $2.3\left(\sigma_{2.3}\right)$ (Lonetto et al. 1992), is thought to act, at least in part, as a sequence-specific, ssDNA-binding element. In this view, $\sigma_{2.3}$ functions by binding the nontemplate strand bases in the melted -10 element, thereby stabilizing the melted state (Roberts and Roberts 1996; Huang et al. 1997; Marr and Roberts 1997). Thinking about $\sigma_{2.3}$ function in this way, the results of Koo et al. (2009c) may seem to present a paradox, since substitution of the primary $\sigma$ melting residues into the alternative $\sigma$ improves melting function on the cognate promoter of the wild-type alternative $\sigma$. However, this can be understood since, in the primary $\sigma$ cognate -10 element, the $-11 \mathrm{~A}$ (TATAAT) is extremely highly conserved (Shultzaberger et al. 2006) and is thought to be the initial base that flips out of the double helix to interact with $\sigma$ and nucleate promoter melting (Lim et al. 2001). Alignment of the $\sigma^{70}, \sigma^{32}$, and $\sigma^{28}$ promoter sequences indicates that the $-11 \mathrm{~A}$ is retained in each of the promoters (see Fig. 1B of Koo et al. 2009c). The conservation of an A at this promoter position for the primary and alternative $\sigma$ promoters; the apparent specificity of the primary $\sigma$ melting residues for this $\mathrm{A}_{\text {; }}$ and the dominant, nucleating effect of this A on the promoter melting process resolves the paradox.

The ability of the primary $\sigma$ melting residues to improve the melting capabilities of the mutant alternative $\sigma s$ was inferred by comparing binding of the mutant $\sigma s$ to double-stranded and fork junction promoter fragments. It will be informative to investigate the mechanisms of melting by the alternative $\sigma \mathrm{s}$ (both mutant and wild type) using the battery of biophysical and biochemical tools that have been brought to bear on the primary $\sigma$ (deHaseth et al. 1998).

While primary os on their own direct transcription from a wide range of poorly conserved promoters, the range of promoters is broadened even further by the influence of trans-acting factors (i.e., transcription activators), which allow transcription from promoters too poor to be used by $\sigma^{70}$ on its own. The influence of trans- acting regulatory factors on the promoter selectivity of holoenzymes containing alternative $\sigma$ s has not been investigated to any extent.

While alternative $\sigma$ factors should clearly know their allowed territory, housekeeping $\sigma \mathrm{s}$ can act on behalf of alternative $\sigma s$ in certain situations. For example, bacterial strains lacking $\sigma^{32}$ can still transcribe heat-shock genes, possibly through the involvement of $\sigma^{70}$ (Zhou et al. $1988)$. It was also shown that the majority of $\sigma^{32}$ promoters can be used by the $\sigma^{70}$ holoenzyme in vivo and in vitro. Some $\sigma^{\mathrm{E}}$ promoters are recognized by $\sigma^{70}$ as well (Wade et al. 2006). The biological role of $\sigma^{70}$ in specific responses, normally thought to be the exclusive purview of alternative $\sigma \mathrm{s}$, remains to be understood.

Alternative $\sigma$ s usually mediate responses to environmental stresses, so these responses need to be rapid, focused, and confined to a tightly defined regulon. The work of Koo et al. (2009c) indicates that this is achieved, at least in part, through the strict promoter recognition requirements of the alternative $\sigma$ s. This is governed by the reduced promoter melting capability of the alternative $\sigma$ s due to the loss of "promoter melting" aromatic residues in $\sigma$ region 2.3 , pointing to an evolutionary tradeoff between promoter melting capability and promoter stringency.

\section{Acknowledgments}

This work was supported by funds from a Merck Post-doctoral Fellowship at The Rockefeller University to A.F., and NIH RO1 GM053759 to S.A.D.

\section{References}

Ades SE. 2008. Regulation by destruction; design of the $\sigma^{\mathrm{E}}$ envelope stress response. Curr Opin Microbiol 11: 535-540.

Barne KA, Bown JA, Busby SJW, Minchin SD. 1997. Region 2.5 of the Escherichia coli RNA polymerase $\sigma^{70}$ subunit is responsible for the recognition of the 'extended -10 ' motif at promoters. $E M B O$ J 16: 4034-4040.

Braun V, Mahren S, Ogierman M. 2003. Regulation of the FecItype ECF $\sigma$ factor by transmembrane signalling. Curr Opin Microbiol 6: 173-180.

Campbell EA, Muzzin O, Chlenov M, Sun JL, Olson CA, Weinman O, Trester-Zedlitz ML, Darst SA. 2002. Structure of the bacterial RNA polymerase promoter specificity $\sigma$ factor. Mol Cell 9: 527-539.

Campbell EA, Tupy JL, Gruber TM, Wang S, Sharp MM, Gross CA, Darst SA. 2003. Crystal structure of Escherichia coli $\sigma^{\mathrm{E}}$ with the cytoplasmic domain of its anti- $\sigma$ RseA. Mol Cell 11: 1067-1078.

Campbell EA, Greenwell R, Anthony JR, Wang S, Lim L, Das K, Sofia HJ, Donohue TJ, Darst SA. 2007. A conserved structural module regulates transcriptional responses to diverse stress signals in bacteria. Mol Cell 27: 793-805.

Chilcott GS, Hughes KT. 2000. Coupling of flagellar gene expression to flagellar assembly in Salmonella enterica serovar typhimurium and Escherichia coli. Microbiol Mol Biol Rev 64: 694-708.

Daniels D, Zuber P, Losick R. 1990. Two amino acids in an RNA polymerase $\sigma$ factor involved in the recognition of adjacent base pairs in the -10 region of a cognate promoter. Proc Natl Acad Sci 87: 8075-8079. 
deHaseth PL, Zupancic ML, Record MTJ. 1998. RNA polymerase-promoter interactions: The comings and going of RNA polymerase. I Bacteriol 180: 3019-3025.

Feklistov A, Barinova N, Sevostyanova A, Heyduk E, Bass I, Vvedenskaya I, Kuznedelov K, Merkiene E, Stavrovskaya E, Klimasauskas S, et al. 2006. A basal promoter element recognized by free RNA polymerase $\sigma$ subunit determines promoter recognition by RNA polymerase holoenzyme. Mol Cell 23: 97-107.

Gardella T, Moyle T, Susskind MM. 1989. A mutant Escherichia coli $\sigma^{70}$ subunit of RNA polymerase with altered promoter specificity. J Mol Biol 206: 579-590.

Gribskov M, Burgess RR. 1986. $\sigma$ Factors from E. coli, B. subtilis, phase SPO1, and phage T4 are homologous proteins. Nucleic Acids Res 14: 6745-6763.

Gruber TM, Bryant DA. 1997. Molecular systematic studies of eubacteria, using $\sigma^{70}$-type $\sigma$ factors of group 1 and group $2 . J$ Bacteriol 179: 1734-1747.

Gruber TM, Gross CA. 2003. Multiple $\sigma$ subunits and the partitioning of bacterial transcription space. Annu Rev Microbiol 57: 441-466.

Guisbert E, Yura T, Rhodius VA, Gross CA. 2008. Convergence of molecular, modeling, and systems approaches for an understanding of the Escherichia coli heat shock response. Microbiol Mol Biol Rev 72: 545-554.

Guo Y, Gralla JD. 1998. Promoter opening via a DNA fork junction binding activity. Proc Natl Acad Sci 95: 1165511660.

Haugen SP, Berkmen MB, Ross W, Gaal T, Ward C, Gourse RL. 2006. rRNA promoter regulation by nonoptimal binding of $\sigma$ region 1.2: An additional recognition element for RNA polymerase. Cell 125: 1069-1082.

Helmann JD, Chamberlin MJ. 1988. Structure and function of bacterial $\sigma$ factors. Annu Rev Biochem 57: 839-872.

Huang X, Lopez de Saro FJ, Helmann JD. 1997. $\sigma$ Factor mutations affecting the sequence-seletive interaction of RNA polymerase with -10 region single-stranded DNA. Nucleic Acids Res 25: 2603-2609.

Juang Y-L, Helmann JD. 1994. A promoter melting region in the primary $\sigma$ factor of Bacillus subtilis: Identification of functionally important aromatic amino acids. I Mol Biol 235: 1470-1488.

Juang Y-L, Helmann JD. 1995. Pathway of promoter melting by Bacillus subtilis RNA polymerase at a stable RNA promoter: Effects of temperature, $\delta$ protein, and $\sigma$ factor mutations. Biochemistry 34: 8465-8473.

Keilty S, Rosenberg M. 1987. Constituitive function of a positively regulated promoter reveals new sequences essential for activity. J Biol Chem 262: 6389-6395.

Klauck E, Typas A, Hengge R. 2007. The $\sigma^{\mathrm{S}}$ subunit of RNA polymerase as a signal integrator and network master regulator in the general stress response in Escherichia coli. Sci Prog 90: 103-127.

Koo B-M, Rhodius VA, Campbell EA, Gross CA. 2009a. Dissection of recognition determinants of Escherichia coli $\sigma^{32}$ suggests a composite -10 region with an 'extended $-10^{\prime}$ motif and a core -10 element. Mol Microbiol 72: 815-829.

Koo B-M, Rhodius VA, Campbell EA, Gross CA. 2009b. Mutational analysis of Escherichia coli $\sigma^{28}$ and its target promoters reveals recognition of a composite -10 regionm, comprised of an 'extended $-10^{\prime}$ motif and a core -10 element. Mol Microbiol 72: 830-843.

Koo B-M, Rhodius VA, Nonaka G, deHaseth PL, Gross CA. 2009c. Reduced capacity of alternative $\sigma$ s to melt promoters ensures stringent promoter recognition. Genes \& Dev (this issue). doi: $10.1101 / \operatorname{gad} .1843709$.
Li W, Stevenson CE, Burton N, Jakimowicz P, Paget MS, Buttner MJ, Lawson DM, Kleanthous C. 2002. Identification and structure of the anti- $\sigma$ factor-binding domain of the disulphide-stress regulated $\sigma$ factor $\sigma^{\mathrm{R}}$ from Stretomyces coelicolor. J Mol Biol 323: 225-236.

Lim HM, Lee HJ, Roy S, Adhya S. 2001. A 'master' in base unpairing during isomerization of a promoter upon RNA polymerase binding. Proc Natl Acad Sci 98: 14849-14852.

Lonetto M, Gribskov M, Gross CA. 1992. The $\sigma^{70}$ family: Sequence conservation and evolutionary relationships. $I$ Bacteriol 174: 3843-3849.

Lozada-Chavez I, Angarica VE, Collado-Vides J, ContrerasMoreira B. 2008. The role of DNA-binding specificity in the evolution of bacterial regulatory networks. I Mol Biol 379: 627-643.

Malhotra A, Severinova E, Darst SA. 1996. Crystal structure of a $\sigma^{70}$ subunit fragment from Escherichia coli RNA polymerase. Cell 87: 127-136.

Marr MT, Roberts JW. 1997. Promoter recognition as measured by binding of polymerase to nontemplate strand oligonucleotide. Science 276: 1258-1260.

Murakami K, Masuda S, Campbell EA, Muzzin O, Darst SA. 2002. Structural basis of transcription initiation: An RNA polymerase holoenzyme/DNA complex. Science 296: 12851290.

Nonaka G, Blankschien M, Herman C, Gross CA, Rhodius VA. 2006. Regulon and promoter analysis of the E. coli heatchock factor, $\sigma^{32}$, reveals a multifaceted cellular response to heat stress. Genes \& Dev 20: 1776-1789.

Paget MS, Helmann JD. 2003. The $\sigma^{70}$ family of $\sigma$ factors. Genome Biol 4: 203. doi: 10.1186/gb-2003-4-1-203.

Pribnow D. 1975. Nucleotide sequence of an RNA polymerase binding site at an early T7 promoter. Proc Natl Acad Sci 72: 784-788.

Roberts CW, Roberts JW. 1996. Base-specific recognition of the nontemplate strand of promoter DNA by E. coli RNA polymerase. Cell 86: 495-501.

Schroeder LA, Gries TJ, Saecker RM, Record MTJ, Harris ME, deHaseth PL. 2009. Evidence for a tyrosine-adenine stacking interaction and for a short-lived open intermediate subsequent to initial binding of Escherichia coli RNA polymerase to promoter DNA. I Mol Biol 385: 339 349.

Shen L, Feng X, Yuan Y, Luo X, Hatch TP, Hughes KT, Liu JS, Zhang YX. 2006. Selective promoter recognition by chlamydial $\sigma^{28}$ holoenzyme. J Bacteriol 188: 7364-7377.

Shultzaberger RK, Chen Z, Lewis KA, Schneider TD. 2006. Anatomy of Escherichia coli $\sigma^{70}$ promoters. Nucleic Acids Res 35: 771-788.

Siegele DA, Hu JC, Walter WA, Gross CA. 1989. Altered promoter recognition by mutant forms of the $\sigma^{70}$ subunit of Escherichia coli RNA polymerase. I Mol Biol 206: 591603.

Sorenson MK, Ray SS, Darst SA. 2004. Crystal structure of the flagellar $\sigma /$ anti- $\sigma$ complex $\sigma^{28} /$ FlgM reveals an intact $\sigma$ factor in an inactive conformation. Mol Cell 14: 127-138.

Stragier P, Parsot C, Bouvier J. 1985. Two functional domains conserved in major and alternate bacterial $\sigma$ factors. FEBS Lett 187: 11-15.

Tatti KM, Jones CH, Moran CPJ. 1991. Genetic evidence for interaction of $\sigma^{\mathrm{E}}$ with the spoIIID promoter in Bacillus subtilis. I Bacteriol 173: 7828-7833.

Tomsic M, Tsujikawa L, Panaghie G, Wang Y, Azok J, deHaseth PL. 2001. Different roles for basic and aromatic amino acids in conserved region 2 of Escherichia coli $\sigma^{70}$ in the nucleation and maintenance of the single-stranded DNA bubble in open 
RNA polymerase-promoter complexes. I Biol Chem 276: 31891-31896.

Tsujikawa L, Tsodikov O, de Haseth P. 2001. Interaction of RNA polymerase with forked DNA: Evidence for two kinetically significant intermediates on the pathway to the final complex. Proc Natl Acad Sci 99: 3493-3498.

Wade JT, Roa DC, Grainger DC, Hurd D, Busby SJ, Struhl K, Nudler E. 2006. Extensive functional overlap between $\sigma$ factors in Escherichia coli. Nat Struct Mol Biol 13: 806-814.

Waldburger C, Gardella T, Wong R, Susskind MM. 1990. Changes in conserved region 2 of Escherichia coli $\sigma^{70}$ affecting promoter recognition. J Mol Biol 215: 267-276.

Zhou YN, Kusukawa N, Erickson JW, Gross CA, Yura T. 1988. Isolation and characterization of Escherichia coli mutants that lack the heat shock $\sigma$ factor $\sigma^{32}$. J Bacteriol 170: 3640 3649. 


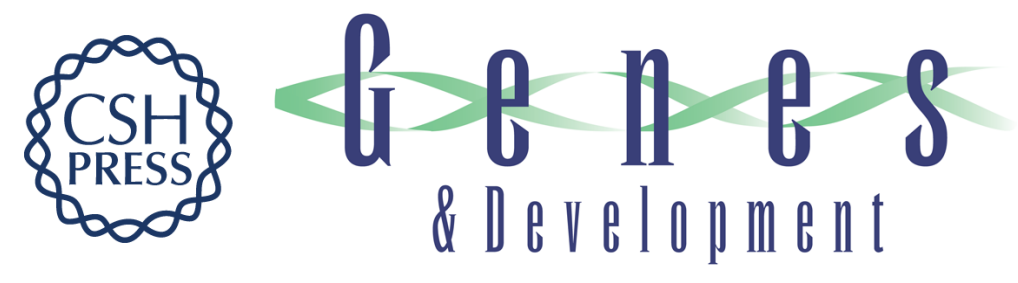

\section{Promoter recognition by bacterial alternative $\sigma$ factors: the price of high selectivity?}

Andrey Feklistov and Seth A. Darst

Genes Dev. 2009, 23:

Access the most recent version at doi:10.1101/gad.1862609
Related Content Reduced capacity of alternative Ãs to melt promoters ensures stringent promoter recognition
Byoung-Mo Koo, Virgil A. Rhodius, Gen Nonaka, et al.
Genes Dev. October , 2009 23: 2426-2436
References This article cites 49 articles, 18 of which can be accessed free at:
http://genesdev.cshlp.org/content/23/20/2371.full.html\#ref-list-1
Articles cited in:
http://genesdev.cshlp.org/content/23/20/2371.full.html\#related-urls

\section{License}
Email Alerting
Service
Receive free email alerts when new articles cite this article - sign up in the box at the top right corner of the article or click here.

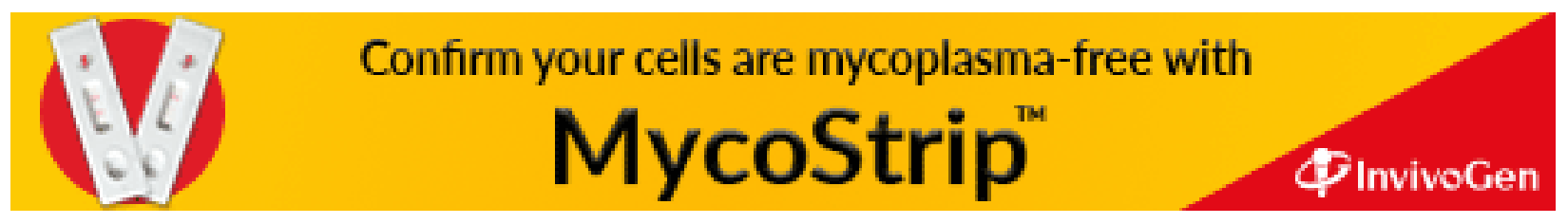

